\title{
Perioperative application of salvianolate on oxidative stress and plasma IMD/ADM2 in patients with acute myocardial infarction undergoing PCI
}

\author{
QIANG ZHANG ${ }^{1}$, FENGJUN JIAO ${ }^{2}$ and CHONGJUN HUA ${ }^{3}$ \\ ${ }^{1}$ Department of Internal Medicine, Dezhou People's Hospital, Dezhou, Shandong 253014; \\ ${ }^{2}$ Department of Cardiovascular Medicine, The First People's Hospital of Xianyang, Xianyang, Shaanxi 712000; \\ ${ }^{3}$ Department of Cardiology, Jinhua Central Hospital, Jinhua, Zhejiang 321000, P.R. China
}

Received June 9, 2016; Accepted January 26, 2017

DOI: $10.3892 /$ etm.2017.4114

\begin{abstract}
The present study was conducted to investigate the effects of salvianolate on perioperative oxidative stress and plasma Intermedin/adrenomedullin 2 (IMD/ADM2) in patients with acute myocardial infarction (AMI) undergoing percutaneous coronary intervention (PCI). The clinical data of 108 patients with AMI treated by PCI were retrospectively analyzed. According to the use of salvianolate in perioperative period, the patients were divided into the study group $(n=62)$ and the control group $(n=46)$. The control group was given routine treatment while the study group was given routine treatment combined with intravenous infusion of salvianolate $(200 \mathrm{mg}$ administered once at $24 \mathrm{~h}$ before operation, once/day after operation, 1 week later, the curative effect was observed). The changes in levels of hemodynamic indexes [fibrinogen (FIB), platelet aggregation rate (PAG), plasma viscosity, packed-cell volume (PCV)], oxidative stress indexes [superoxide dismutase (SOD), glutathione peroxidase (GSH Px)], cardiac function indexes [left ventricular ejection fraction (LVEF), cardiac output (CO)], related biochemical indexes [high-sensitivity $\mathrm{C}$-reactive protein (hs-CRP), N-terminal pro brain natriuretic peptide (NT proBNP)] and plasma IMD/ADM2 in the two groups at $24 \mathrm{~h}$ pre-operation and the 8th day postoperation were analyzed. On the 8th day post-operation, hemodynamic indexes such as FIB (3.3 \pm 1.0 and 3.9 \pm 0.9$) \mathrm{g} / 1$, PAG $(20.6 \pm 6.5$ and $41.6 \pm 7.7) \%$, plasma viscosity $(1.1 \pm 0.5$ and $1.7 \pm 0.6) \mathrm{mPa} \bullet \mathrm{s} / 120 \mathrm{~s}^{-1}$ and PCV $(40.0 \pm 3.8$ and $45.5 \pm 3.7) \%$, the related biochemical
\end{abstract}

Correspondence to: Dr Chongjun Hua, Department of Cardiology, Jinhua Central Hospital, 351 Bright Moon Street, Jinhua, Zhejiang 321000, P.R. China

E-mail: huachongjun2016@163.com

Key words: salvianolate, acute myocardial infarction, percutaneous coronary intervention, perioperative period, oxidative stress, IMD/ADM2 indexes such as hs-CRP $(55.1 \pm 4.8$ and $79.3 \pm 5.3) \mathrm{mg} / \mathrm{l}$ and NT-proBNP $(435.6 \pm 305.2$ and $788.6 \pm 310.8) \mathrm{ng} / \mathrm{l}$ in the two groups were significantly decreased compared to those pre-operation; the differences were statistically significant $(\mathrm{P}<0.05)$. The oxidative stress indexes such as SOD $(43.6 \pm 1.9$ and 24.2 \pm 2.0$) \mathrm{U} / \mathrm{ml}$ and GSH-Px $(822.6 \pm 13.52$ and $742.6 \pm 62.6)$ enzyme activity unit, the cardiac function indexes such as LVEF $(45.1 \pm 3.5$ and $41.9 \pm 3.3) \%$ and $\mathrm{CO}(4.6 \pm 0.5$ and $4.1 \pm 0.5) \mathrm{l} / \mathrm{min}$ and plasma IMD/ADM2 $(163.5 \pm 20.2$ and $144.2 \pm 22.5) \mathrm{pg} / \mathrm{ml}$ in the two groups were significantly increased compared to those pre-operation; the differences were statistically significant $(\mathrm{P}<0.05)$. In conclusion, applying intravenous infusion of salvianolate in patients with AMI during perioperative period of PCI can effectively improve the oxidative stress reaction and have positive effects on regulating plasma IMD/ADM2 level, which promoted cardiac function recovery and enhances myocardial perfusion volume.

\section{Introduction}

According to previous literature, the incidence of acute myocardial infarction (AMI) in China tends to increase gradually year by year, with an estimated $>500,000$ new AMI cases each year (1). Its high incidence and mortality rates have caught extensive attention of clinicians and society. The way to improve the clinical curative effect on AMI and reduce the risk of death through scientific and effective curative methods has become a hot topic of investigation. With salvianolic acid as its main active component, salvianolate is the purified extract of saliva and can activate blood and disperse stagnation (2). When applied in the clinical treatment of AMI, salvianolate has positive effects on promoting the recovery of myocardial perfusion volume, relieving myocardial blood microcirculation and improving lipid peroxidation (3). An increasing number of scholars have found that Intermedin/adrenomedullin 2 (IMD/ADM2) can exert its effect together with the common receptor of calcitonin gene-related peptide (CGRP) superfamily and play a positive role in regulating the homeostasis of cardiovascular and respiratory system (4). The higher the plasma IMD/ADM2 level is, the greater its effect on 
cardiovascular protection (5). At present, there are quite a few clinical reports on the effect of salvianolate on the hemodynamic state and myocardial damage of patients with AMI, but few studies have conducted extensive investigations on its effect on the plasma IMD/ADM2 of the subjects. As a result, this study retrospectively analyzed the clinical data of 108 patients with AMI treated by percutaneous coronary intervention (PCI), compared the oxidative stress indexes, inflammatory indexes, cardiac function indexes, hemodynamic indexes and plasma IMD/ADM2 level in the two groups and investigated the effects of perioperative application of salvianolate on promoting cardiac function recovery and improving its prognostic quality. The present study was conducted in order to seek more effective approaches to provide a basis for improving the postoperative recovery of patients with AMI treated by PCI.

\section{Materials and methods}

Data source. The clinical data of 108 patients with AMI treated by PCI from October 2012 to April 2014 in our hospital were retrospectively analyzed. Inclusion criteria were as follows: i) Patients who met relevant AMI diagnostic criteria according to American College of Cardiology/American Heart Association (6,7); ii) patients who met relevant treatment indications for PCI; iii) patients who were classified as Killip II-III; iv) patients with time window $<6 \mathrm{~h}$. Exclusion criteria were as follows: i) Patients complicated with infectious disease, severe autoimmune disease, hemorrhagic disease, coagulation disorder, severe organ dysfunction syndrome or malignant tumor; ii) patients complicated with mental diseases or consciousness disturbance; iii) patients with allergic constitutions or relevant treatment contraindications; iv) patients who were $>80$ years old; v) pregnant or lactating women; vi) patients who were classified as Killip I or IV; vii) patients who were dead or transferred or whose therapeutic schedules were changed during perioperative period or who were out of touch during follow-up period.

According to the use of salvianolate in perioperative period, 108 patients were divided into the study group $(n=62)$ and the control group $(n=46)$. The study group included 39 males and 23 females, aged from 45-79 years, with an average of $(66.4 \pm 8.2)$ years. Time window was $2.2 \pm 1.0 \mathrm{~h}$. A total of 42, 21 and 38 cases, respectively, were complicated with hypertension, diabetes and hyperlipidemia; 25 cases were complicated with family heredity history. The control group included 29 males and 17 females, aged from $44-80$ years, with an average of $66.7 \pm 8.0$ years. Time window was $2.3 \pm 1.1 \mathrm{~h}$. A total of 32,18 and 27 cases, respectively, were complicated with hypertension, diabetes and hyperlipidemia; 20 cases with family heredity history. Basic data of the two above groups were of no statistical significance $(\mathrm{P}>0.05)$ and comparable.

Curative methods. Patients in the two groups were all treated by PCI according to the Guidelines of American College of Cardiology/American Heart Association (6). Patients were administered oral medication of $300 \mathrm{mg}$ clopidogrel $+300 \mathrm{mg}$ aspirin before operation and atorvastatin calcium tablets, aspirin and clopidogrel after operation, and administered nitrates, $\beta$ receptor blocker and angiotensin converting enzyme inhibitor as ordered by doctors according to the severity of the illness. The study group was given the above-mentioned treatment combined with intravenous infusion of salvianolate. Salvianolate (200 mg, license no. NMPN: Z20050248; Shanghai Green Valley Pharmaceutical Co., Ltd., Shanghai, China), was administered intravenously once at $24 \mathrm{~h}$ before operation, once/day after operation, administered on the day of operation, 1 week later, the curative effect was observed.

Indexes of detection methods. From each patient $5 \mathrm{ml}$ morning fasting antecubital venous blood was drawn at $24 \mathrm{~h}$ before operation and the 8th day after operation, and the high-sensitivity C-reactive protein (hs-CRP) level was detected by ELISA according to the operation instruction of hs-CRP kit (Shanghai Xinyu Biotechnology Co., Ltd., Shanghai, China), the NT-proBNP level was detected by colloidal gold method according to the operation instruction of NT-proBNP kit (Hangzhou Puwang Biotechnology Co., Ltd., Hangzhou, China), the superoxide dismutase (SOD) level was detected by xanthine oxidase method according to the operation instruction of SOD kit (Shanghai Solarbio Biotechnology Co., Ltd., Shanghai, China), the GSH-Px level was detected by colorimetric method according to the operation instruction of GSH-Px kit (Shanghai Huzheng Biotechnology Co., Ltd., Shanghai, China), the plasma IMD/ADM2 level was detected by radioimmunoassay according to the operation instruction of IMD/ADM2 kit (Phoenix Pharmaceutical Inc., St. Joseph, MO, USA). Cardiac function indexes such as left ventricular ejection fraction (LVEF) and cardiac output (CO) were detected by diasonograph (ACUSON X300; Siemens AG, Munich, Germany). The plasma viscosity was detected by blood viscometer (LG R 80 series; Beijing Zhongqinshidi Scientific Apparatus Co., Ltd., Beijing, China). Fibrinogen (FIB) was detected by automated coagulation analyzer (ACL7000; Beckman Coulter, Inc., Brea, CA, USA). Platelet aggregation rate (PAG) level was detected by kinetic turbidimetry method. Packed-cell volume (PCV) level was detected by Wen's method.

Observation indexes. Clinical data from the two groups were retrospectively analyzed. The changes in levels of hemodynamic indexes (FIB, PAG, plasma viscosity, PCV), oxidative stress indexes [SOD, glutathione peroxidase (GSH Px)], cardiac function indexes (LVEF and CO), related biochemical indexes (hs-CRP, N-terminal pro brain natriuretic peptide) and plasma IMD/ADM2 at $24 \mathrm{~h}$ before operation and the 8th day after operation were compared.

Statistical processing. SPSS 19.0 (SPSS, Inc., Chicago, IL, USA) statistical software was used for recording and analysis. The data are expressed as mean \pm standard deviation, and evaluated by t-test. $\mathrm{P}<0.05$ was considered to indicate a statistically significant difference.

\section{Results}

Comparison of hemodynamic indexes between the two groups before and after treatment. On the 8th day postoperation, hemodynamic indexes such as FIB, PAG, plasma viscosity and 
Table I. Comparison of hemodynamic indexes between the two groups before and after treatment (mean \pm standard deviation).

\begin{tabular}{|c|c|c|c|c|c|c|}
\hline Group & Cases & Observation time & FIB, g/l & $\mathrm{PAG}, \%$ & Plasma viscosity $\mathrm{mPa} \bullet \mathrm{s} / 120 \mathrm{~s}^{-1}$ & $\mathrm{PCV}, \%$ \\
\hline Study & 62 & $\begin{array}{l}24 \mathrm{~h} \text { before operation } \\
\text { the } 8 \text { th day after operation }\end{array}$ & $\begin{array}{l}4.2 \pm 1.9 \\
3.3 \pm 1.0^{\mathrm{a}, \mathrm{b}}\end{array}$ & $\begin{array}{l}87.9 \pm 16.5 \\
20.6 \pm 6.5^{\mathrm{a}, \mathrm{b}}\end{array}$ & $\begin{array}{l}2.1 \pm 0.9 \\
1.1 \pm 0.5^{\mathrm{a}, \mathrm{b}}\end{array}$ & $\begin{array}{l}49.5 \pm 3.9 \\
40.0 \pm 3.8^{\mathrm{a}, \mathrm{b}}\end{array}$ \\
\hline Control & 46 & $\begin{array}{l}24 \mathrm{~h} \text { before operation } \\
\text { the } 8 \text { th day after operation }\end{array}$ & $\begin{array}{l}4.2 \pm 1.9 \\
3.9 \pm 0.9^{\mathrm{a}}\end{array}$ & $\begin{array}{l}87.7 \pm 16.3 \\
41.6 \pm 7.7^{\mathrm{a}}\end{array}$ & $\begin{array}{l}2.2 \pm 0.9 \\
1.7 \pm 0.6^{\mathrm{a}}\end{array}$ & $\begin{array}{l}49.6 \pm 3.8 \\
45.5 \pm 3.7^{\mathrm{a}}\end{array}$ \\
\hline
\end{tabular}

${ }^{a} \mathrm{P}<0.05$, compared with that in the same group before operation; ${ }^{\mathrm{b}} \mathrm{P}<0.05$, compared with that in the control group on the 8 th day after operation.

Table II. Comparison of oxidative stress indexes between the two groups before and after treatment (mean \pm standard deviation).

\begin{tabular}{lcllr}
\hline Group & Cases & \multicolumn{1}{c}{ Observation time } & SOD, U/ml & GSH-Px, enzyme activity unit \\
\hline Study & 62 & 24 h before operation & $16.8 \pm 1.8$ & $612.8 \pm 133.5$ \\
& & the 8th day after operation & $43.6 \pm 1.9^{\mathrm{a}, \mathrm{b}}$ & $822.6 \pm 135.2^{\mathrm{a}, \mathrm{b}}$ \\
Observation & 46 & 24 h before operation & $16.7 \pm 1.9$ & $614.4 \pm 132.9$ \\
& & the 8th day after operation & $24.2 \pm 2.0^{\mathrm{a}}$ & $742.6 \pm 62.6^{\mathrm{a}}$ \\
\hline
\end{tabular}

${ }^{\mathrm{a}} \mathrm{P}<0.05$, compared with that in the same group before operation; ${ }^{\mathrm{b}} \mathrm{P}<0.05$, compared with that in the control group on the 8 th day after operation.

Table III. Comparison of cardiac function indexes between the two groups before and after treatment (mean \pm standard deviation).

\begin{tabular}{lclll}
\hline Group & Cases & \multicolumn{1}{c}{ Observation time } & LVEF, $\%$ & CO, 1/min \\
\hline Study & 62 & 24 h before operation & $35.9 \pm 5.9$ & $3.7 \pm 0.6$ \\
& & the 8 th day after operation & $45.1 \pm 3.5^{\mathrm{a}, \mathrm{b}}$ & $3.6 \pm 0.5^{\mathrm{a}, \mathrm{b}}$ \\
Control & 46 & $24 \mathrm{~h}$ before operation & $36.0 \pm 6.0$ & $3.6 \pm 0.7$ \\
& & the 8th day after operation & $41.9 \pm 3.3^{\mathrm{a}}$ & $4.1 \pm 0.5^{\mathrm{a}}$ \\
\hline
\end{tabular}

${ }^{\mathrm{a}} \mathrm{P}<0.05$, compared with that in the same group before operation; ${ }^{\mathrm{b}} \mathrm{P}<0.05$, compared with that in the control group on the 8th day after operation.

PCV in the two groups were significantly decreased compared with those before operation; the differences were statistically significant $(\mathrm{P}<0.05)$ (Table I).

Comparison of oxidative stress indexes between the two groups before and after treatment. On the 8th day postoperation, oxidative stress indexes such as SOD and GSH-Px in the two groups were significantly increased compared with those pre-operation; the differences were statistically significant $(\mathrm{P}<0.05)$ (Table II).

Comparison of cardiac function indexes between the two groups before and after treatment. On the 8th day postoperation, cardiac function indexes such as LVEF and CO in the two groups were significantly increased compared to those pre-operation; the differences were statistically significant $(\mathrm{P}<0.05)$ (Table III).

Comparison of related biochemical indexes between the two groups before and after treatment. On the 8th day postoperation, the level of hs-CRP and NT-proBNP in the two groups were significantly decreased compared to those pre-operation; the differences were statistically significant $(\mathrm{P}<0.05)$ (Table IV).

Comparison of plasma IMD/ADM2 level between the two groups before and after treatment. On the 8th day postoperation, plasma IMD/ADM2 level in the two groups were significantly increased compared to those pre-operation; the differences were statistically significant $(\mathrm{P}<0.05)$ (Table V).

\section{Discussion}

AMI is a common clinical cardiovascular disease with high mortality rates and great influence on the health, safety, prognosis and quality of life of patients. At present, there are numerous clinical reports on the pathogenesis of AMI. Overwork, emotional excitement, overeating, cold and hot stimulation, long-term alcohol abuse or smoking can increase the risk of AMI, which needs clinical attention. Currently, PCI is one of the most important clinical operations that can rebuild blood supply of patients with AMI $(7,8)$. PCI has positive effects on shrinking myocardial infarction areas, promoting the recovery of myocardial blood supply, relieving 
Table IV.Comparison of related biochemical indexes between the two groups before and after treatment (mean \pm standard deviation).

\begin{tabular}{|c|c|c|c|c|}
\hline Group & Cases & Observation time & hs-CRP, mg/l & NT-proBNP, ng/l \\
\hline Study & 62 & $\begin{array}{l}24 \mathrm{~h} \text { before operation } \\
\text { the } 8 \text { th day after operation }\end{array}$ & $\begin{array}{l}96.2 \pm 10.3 \\
55.1 \pm 4.8^{\mathrm{a}, \mathrm{b}}\end{array}$ & $\begin{array}{c}1063.5 \pm 519.3 \\
435.6 \pm 305.2^{\mathrm{a}, \mathrm{b}}\end{array}$ \\
\hline Control & 46 & $\begin{array}{l}24 \mathrm{~h} \text { before operation } \\
\text { the } 8 \text { th day after operation }\end{array}$ & $\begin{array}{l}96.0 \pm 10.3 \\
79.3 \pm 5.3^{\mathrm{a}}\end{array}$ & $\begin{array}{c}1065.7 \pm 520.5 \\
788.6 \pm 310.8^{\mathrm{a}}\end{array}$ \\
\hline
\end{tabular}

${ }^{\mathrm{a}} \mathrm{P}<0.05$, compared with that in the same group before operation; ${ }^{\mathrm{b}} \mathrm{P}<0.05$, compared with that in the control group on the 8 th day after operation.

Table V. Comparison of plasma IMD/ADM2 level between the two groups before and after treatment (mean \pm standard deviation, $\mathrm{pg} / \mathrm{ml})$.

\begin{tabular}{lccc}
\hline Group & Cases & $\begin{array}{c}\text { Before } \\
\text { operation }\end{array}$ & $\begin{array}{c}\text { The 8th day } \\
\text { after operation }\end{array}$ \\
\hline Study & 62 & $127.6 \pm 30.5$ & $163.5 \pm 20.2^{\mathrm{a}, \mathrm{b}}$ \\
Control & 46 & $128.0 \pm 30.3$ & $144.2 \pm 22.5^{\mathrm{a}}$ \\
\hline
\end{tabular}

${ }^{\mathrm{a}} \mathrm{P}<0.05$, compared with that before operation; ${ }^{\mathrm{b}} \mathrm{P}<0.05$, compared with that in the control group.

ischemia and anoxia (9). Relevant studies have shown that the epicardial-vascular blood perfusion volume of most patients with AMI gradually recovered after treatment of PCI, which may increase the risk of thrombus detachment or distant embolism of microcirculation (10), damage the microcirculation after treatment, affect cardiovascular reflow, increase the probability of re-infarction and harm to the prognosis recovery of patients. Methods to promote the recovery of hemodynamic indexes after treatment of PCI and regulate the oxidative stress response of the human body through effective curative methods has become an important investigation field.

As main active component of the Chinese medicinal material salvia, salvianolate is a kind of salt compound whose main constituents are salvia magnesium acetate and magnesium lithospermate B (11). It is known to exert multiple effects on activating blood, dispersing stagnation, anti-oxidation, anti-platelet aggregation, scavenging free radical and improving blood microcirculation (12). It has also been widely used in clinical treatment of multiple cardiovascular and cerebrovascular diseases. The present study retrospectively analyzed the clinical data of 62 patients treated by intravenous infusion of salvianolate during perioperative period of PCI and found that on the 8th day postoperation, cardiac function indexes such as LVEF and $\mathrm{CO}$ in the two groups were significantly increased compared with those before operation. These results showed that intravenous infusion of salvianolate peri-operatively is more beneficial for the cardiac function recovery of patients and has positive effects on improving myocardial perfusion volume and promoting the recovery of CO. Fan et al (13) also acknowledged the conclusions mentioned above in their reports, and they believed that $>80 \%$ of salvianolate is salvia magnesium acetate, which can play an important role in expanding cardiovascular blood flow, inhibiting the synthesis of endothelin, and help improve myocardial ischemia and anoxia to promote a positive outcome.

Apart from cardiac function, the effects of salvianolate on the oxidative stress response, inflammatory response and the changes in levels of hemodynamic indexes in perioperative period of patients with AMI were also analyzed in the study. We found that the plasma viscosity, FIB level, PCV and PAG in the patients in the study group, who used salvianolate during perioperative period, were all lower compared to those in the control group. These results are consistent with the conclusion of Yang et al (14). This confirms that the peri-operative application of salvianolate can effectively reduce plasma viscosity, facilitate fibrinolysis, accelerate thrombolysis and inhibit re-thrombosis post-operationally by activating plasminogen to promote the recovery of patients with AMI. In addition, the function of anti-oxidation and scavenging free radical can also help regulate the oxidative stress response of the human body and increasing the SOD level post-operation so as to exert SOD's function of regulating blood lipid metabolism and anti-aging, doubly improve the hemodynamic state during the perioperative period to reduce the risk of re-infarction postoperation. As a common clinical inflammatory marker of AMI and an important risk factor for cardiovascular diseases, hp-CRP is a type of highly-sensitive, non-specific acute phase reaction protein which can correctly reflect the inflammatory status. After PCI, the hp-CRP level in the study group was significantly lower than that in the control group, showing that salvianolate can regulate the systemic inflammation to some degree which may be related with the spontaneous reduction of systemic inflammation after the improvement of cardiac function and the recovery of hemodynamic state.

IMD/ADM2, whose structural function is similar to adrenomedullin, is a new member of CGRP superfamily and can ideally protect the cardiovascular system in cardiovascular disease (15). Previous study has demonstrated that IMD/ADM2 is closely related with the incidence, development and outcome of coronary heart disease (16); although its structure is similar to AMD, the mechanism of action and the physiopathological significance of the two have obvious differences (17), which should be taken seriously. The present study also places emphasis on the influence of salvianolate on the plasma IMD/ADM2 level of patients with AMI during PCI peri-operative period, and found that the plasma IMD/ADM2 level of the patients with AMI was significantly decreased compared to the 
normal value, showing that the protective effect of human body on the cardiovascular system was weakened. Furthermore, the study found that the plasma IMD/ADM2 level post-treatment of PCI and intravenous infusion of salvianolate was significantly increased compared to that before treatment and than that in the control group. This confirms that the application of salvianolate during PCI perioperative period can regulate the IMD/ADM2 level of human body to some degree. After the medicine, the oxidative stress indexes, the status of blood lipid metabolism and hemodynamic status were all significantly improved compared to those before treatment. This was beneficial for relieving cardiovascular damage and promoting the recovery of the function of cardiovascular system.

Apart from above-mentioned conclusions, Liu et al (18) included some other clinical application methods of salvianolate into the research, and found that it can not only exert positive effects on cardiovascular and cerebrovascular diseases, but also achieve antitumor effect in vitro by functioning in cell cycle and inducing tumor cell apoptosis. This mechanism can be further investigated as another clinical feasible direction of therapy of salvianolate. Limited by the number of samples, the present study did not analyze the effect of salvianolate on inhibiting tumors in cancer patients by listing patients with AMI as key research subjects. As a result, the accuracy of the conclusions mentioned above is hard to evaluate, but it can be further analyzed as a follow-up research topic after enrolling more patients.

The present study found that applying intravenous infusion of salvianolate in patients with AMI during perioperative period of PCI can not only promote the recovery of disease of patients with AMI, improve the hemodynamic status and accelerate the recovery of myocardial microcirculation, but also regulate the oxidative stress reactions of human body, reduce inflammatory responses and improve the plasma IMD/ADM2 level. However, due to the limited quantity of samples and the time of follow-up visits, certain parts of the present study are significantly different from other reports, which can be further analyzed as follow-up after enlarging the quantity of samples.

In conclusion, applying intravenous infusion of salvianolate in patients with AMI during perioperative period of PCI can effectively improve the oxidative stress reactions, have positive effects on regulating plasma IMD/ADM2 level, promote cardiac function recovery, enhance myocardial perfusion volume, and help the recovery of the patients.

\section{References}

1. Xie D, Li W, Wang Y, Gu H, Teo K, Liu L and Yusuf S; INTERHEART China study Investigators: Sleep duration, snoring habits and risk of acute myocardial infarction in China population: Results of the INTERHEART study. BMC Public Health 14: 531, 2014.

2. Yang J, Zhang G, Tian J, Li C, Jiang W, Xing Y, Zhu H, Hou J, $\mathrm{Xu} \mathrm{H}$ and Wu J: Cardioprotective effect of SMND-309, a novel derivate of salvianolic acid $\mathrm{B}$ on acute myocardial infarction in rats. Basic Clin Pharmacol Toxicol 106: 317-323, 2010.

3. Wang X, Wang Y, Jiang M, Zhu Y, Hu L, Fan G, Wang Y, Li X and Gao X: Differential cardioprotective effects of salvianolic acid and tanshinone on acute myocardial infarction are mediated by unique signaling pathways. J Ethnopharmacol 135: 662-671, 2011.
4. Holmes D, Campbell M, Harbinson M and Bell D: Protective effects of intermedin on cardiovascular, pulmonary and renal diseases: Comparison with adrenomedullin and CGRP. Curr Protein Pept Sci 14: 294-329, 2013.

5. Huang CY, Chen SY, Fu RH, Huang YC, Chen SY, Shyu WC, Lin SZ and Liu SP: Differentiation of embryonic stem cells into cardiomyocytes used to investigate the cardioprotective effect of salvianolic acid B through BNIP3 involved pathway. Cell Transplant 24: 561-571, 2015.

6. Antman EM, Anbe DT, Armstrong PW, Bates ER, Green LA, Hand M, Hochman JS, Krumholz HM, Kushner FG, Lamas GA, et al; American College of Cardiology; American College of Cardiology/American Heart Association Task Force on Practice Guidelines (Writing Committee to Revise the 1999 Guidelines for the Management of Patients With Acute Myocardial Infarction): ACC/AHA guidelines for the management of patients with ST-elevation myocardial infarction - executive summary: a report of the American College of Cardiology/American Heart Association Task Force on Practice Guidelines (Writing Committee to Revise the 1999 Guidelines for the Management of Patients With Acute Myocardial Infarction). Circulation 110: 588-636, 2004.

7. Gillum RF, Fortmann SP, Prineas RJ and Kottke TE: International diagnostic criteria for acute myocardial infarction and acute stroke. Am Heart J 108: 150-158, 1984.

8. Gheeraert PJ, De Buyzere ML, Taeymans YM, Gillebert TC, Henriques JP, De Backer G and De Bacquer D: Risk factors for primary ventricular fibrillation during acute myocardial infarction: A systematic review and meta-analysis. Eur Heart J 27: 2499-2510, 2006.

9. Dangas GD, Caixeta A, Mehran R, Parise H, Lansky AJ, Cristea E, Brodie BR, Witzenbichler B, Guagliumi G, Peruga JZ, et al; Harmonizing Outcomes with Revascularization and Stents in Acute Myocardial Infarction (HORIZONS-AMI) Trial Investigators: Frequency and predictors of stent thrombosis after percutaneous coronary intervention in acute myocardial infarction. Circulation 123: 1745-1756, 2011.

10. Lindsey JB, Marso SP, Pencina M, Stolker JM, Kennedy KF, Rihal C, Barsness G, Piana RN, Goldberg SL, Cutlip DE, et al; EVENT Registry Investigators: Prognostic impact of periprocedural bleeding and myocardial infarction after percutaneous coronary intervention in unselected patients: Results from the EVENT (evaluation of drug-eluting stents and ischemic events) registry. JACC Cardiovasc Interv 2: 1074-1082, 2009.

11. Zhang HS and Wang SQ: Salvianolic acid B from Salvia miltiorrhiza inhibits tumor necrosis factor-alpha(TNF-alpha)-induced MMP-2 upregulation in human aortic smooth muscle cells via suppression of $\mathrm{NAD}(\mathrm{P}) \mathrm{H}$ oxidase-derived reactive oxygen species. J Mol Cell Cardiol 41: 138-148, 2006.

12. Zhou L, Zuo Z and Chow MS: Danshen: An overview of its chemistry, pharmacology, pharmacokinetics, and clinical use. J Clin Pharmacol 45: 1345-1359, 2005.

13. Fan H, Yang L, Fu F, Xu H, Meng Q, Zhu H, Teng L, Yang M, Zhang L, Zhang Z and Liu K: Cardioprotective effects of salvianolic acid a on myocardial ischemia-reperfusion injury in vivo and in vitro. Evid Based Complement Alternat Med 2012: 508938, 2012.

14. Yang Q, Wang S, Xie Y, Wang J, Li H, Zhou X and Liu W: Effect of salvianolic acid B and paeonol on blood lipid metabolism and hemorrheology in myocardial ischemia rabbits induced by pituitruin. Int J Mol Sci 11: 3696-3704, 2010.

15. Burak Kandilci H, Gumusel B, Wasserman A, Witriol N and Lippton $\mathrm{H}$ : Intermedin/adrenomedullin-2 dilates the rat pulmonary vascular bed: Dependence on CGRP receptors and nitric oxide release. Peptides 27: 1390-1396, 2006.

16. Yang JH, Qi YF, Jia YX, Pan CS, Zhao J, Yang J, Chang JK and Tang CS: Protective effects of intermedin/adrenomedullin 2 on ischemia/reperfusion injury in isolated rat hearts. Peptides 26: 501-507, 2005

17. Pfeil U, Aslam M, Paddenberg R, Quanz K, Chang CL, Park JI, Gries B, Rafiq A, Faulhammer P, Goldenberg A, et al: Intermedin/adrenomedullin-2 is a hypoxia-induced endothelial peptide that stabilizes pulmonary microvascular permeability. Am J Physiol Lung Cell Mol Physiol 297: L837-L845, 2009.

18. Liu CL, Xie LX, Li M, Durairajan SS, Goto S and Huang JD: Salvianolic acid B inhibits hydrogen peroxide-induced endothelial cell apoptosis through regulating PI3K/Akt signaling. PLoS One 2: e1321, 2007. 